\title{
Relative influence of model assumptions and measurement procedures in the analysis of the MEG
}

\author{
$\begin{array}{lll}\text { J.W. H. Meijs } & \text { M. J. Peters } & \text { H. B. K. Boom }\end{array}$ \\ Department of Electrical Engineering. Twente University, PO Box 217, 7500 AE Enschede, The Netherlands

\section{F. H. Lopes da Silva} \\ Biological Centre, University of Amsterdam, Kruislaan 320, Amsterdam, The Netherlands
}

\begin{abstract}
The relative influences of several model parameters and measurement setups on the MEG are studied quantitatively. The influences of the number of grid points of the flux transformer and the accuracy of the measurements are analysed using as criterion a relative difference measure (RDM). In a similar way, using the RDM, the influence of various models is evaluated. The volume conductor, i.e. the head, is described by three different compartment models: the first model consists of concentric spheres, the second of eccentric spheres optimally fitting the individual compartments of the head, and the third consists of realistically shaped compartments. The evaluation of the influence of the model of the source on the MEG is studied by taking either one single current dipole or a set of two current dipoles. The RDM described in the paper is shown to be a valuable measure in the quantitative analysis of MEGs.
\end{abstract}

Keywords-Experimental accuracy, Grid point density, Magnetoencephalogram. Source model, Volume conductor model

Med. \& Biol. Eng. \& Comput., 1988, 26, 136-142

\section{Introduction}

ONE OF THE MOST promising applications of the magnetoencephalogram (MEG) concerns the estimation of sources of activity in the brain. To determine the location, strength and orientation of the source from externally observed magnetic fields, both the source and the volume conductor have to be modelled. The magnetic field distributions obtained by forward calculations are usually presented as isofunction maps, as are MEGs measured at different points outside the head. In general, measured and calculated maps will show differences due, for instance, to measurement errors and model discrepancies. Therefore, it is useful to have a measure which allows us to quantify differences between two isofield maps. Several ways of obtaining such a measure have been proposed. Some authors merely compared the extreme values of the maps (Meiss et al., 1987a); others defined and compared an error parameter (WEINBERG et al., 1985; STOK, 1987). This error parameter is defined as the difference between an initial (known) source and the best fitting equivalent dipole, but can only be computed if an inverse procedure is available. Others used eigenfunction analysis (MACAULAY et al., 1985) or spatial Fourier analysis (LeHMANN, 1983) for this purpose. However, all these measures have one or more disadvantages: their value may strongly depend on the number and/or location of the measurement points, the necessary complex inverse techniques are not often available or the measure only deals with one specific aspect of the field being studied (e.g. spatial frequencies, eigenvalues).

First received 30th May and in final form 22nd September 1987 (C) IFMBE: 1988
Our aim in the present study is to investigate under which conditions differences in cerebral magnetic field distributions can be quantitatively assessed using a measurement which does not involve the disadvantages mentioned above. This is of practical importance to be able to decide which source or volume conductor models are most suitable to account for a given set of experimental measures. We propose that the relative difference measure $(R D M)$ is appropriate to quantify differences between two maps. The $R D M$ is defined as the squared differences of two fields integrated over the recording surface. The $R D M$ does not depend on the location of the measurement points nor are inverse techniques required. This measurement does not depend on a specific model assumption and it can be used for the analysis of any set of field maps.

For example, if we have environmental noise which provides a large contribution to the $R D M$ when compared with a map without noise, there is no need to reduce the $R D M$ due to the discrete sampling of the signal if its value is a factor of 10 lower than the noise contribution. On the other hand, if we find that the use of an improved source model results in an $R D M$ which has the same order of magnitude as the value of the $R D M$ due to a certain measuring procedure, we should use this improved source model in the analysis of the measurements.

\section{Methods}

The relative difference measure used to compare two maps is defined as

$$
R D M=\sqrt{\left(\frac{\int_{S}\left(F_{r}-F\right)^{2} d S}{\int_{S} F_{r}^{2} d S}\right)}
$$


where

$F_{r}$ is the reference field

$F$ is the field distribution obtained under conditions which differ from $F_{r}$ in one aspect only

$S$ is the surface area of the map considered.

If not stated otherwise, the reference field $F_{r}$ is chosen to be the radial component of the magnetic field generated by a current dipole situated in a concentric multishell spheres model. The recording sphere is concentric with this multishell sphere representing the head. For the reference field this implies that neither the volume currents nor radially oriented dipoles contribute to it. In fact the reference field is identical to the field generated by a dipole in an infinite homogeneous isotropic medium (GRYNSZPAN and GESELOWITZ, 1973). The size of sector $S$ of the recording sphere is chosen such that the solid angle is constant with respect to different source locations and that the extremes are incorporated.

The $N$ grid points taken are projected on a plane so that the distances in both the $e_{\theta}$ and the $e_{\phi}$ direction are conserved. The field values at these $N$ projected grid points are interpolated yielding function values in a fine regular grid of $20 \times 20$ points, using a fifth degree bivariate interpolation technique (AKIMA, 1978). The surface integrals are computed numerically from this fine grid of interpolated function values. An important feature of this technique is the fact that all field distributions are expressed in the same 400 points, making it possible to compare two different field patterns even in cases where the original grid points do not coincide. The reference field is calculated in so many points as necessary for the map of this field to show an ideal dipole pattern, as illustrated in Fig. $1 a$ (in this case $N=400$ ).

The parameters used to compute $F$ are changed one at a time with respect to the parameters used to compute $F_{r}$.
The influence of these changes on the map is given by the associated $R D M$ value.

(a) To estimate the influence of the number of grid points on the field pattern, $F$ is calculated as a function of $N$ where $N$ is reduced systematically from 100 $(=10 \times 10)$ down to $16(=4 \times 4)$ points. When a signal is sampled in a number of points, an error is introduced which is due to the fact that the spatial Fourier spectrum is deformed (ROMANI and LEONI, 1984).

(b) To evaluate the influence of the gradiometer on the field pattern, the procedure of reducing $N$ is repeated by taking the effective magnetic field $B_{e f f}$ instead of a magnetic field component for both $F$ and $F_{r} . B_{e f f}$ is the net magnetic flux through the gradiometer divided by the area of the pickup coil, and is defined as the field value at the centre of the pickup coil. In our computations a symmetric second-order gradiometer with a baseline of $50 \mathrm{~mm}$ and a coil diameter of $30 \mathrm{~mm}$ is used. The computation of the flux through the coil is carried out numerically by approximating the surface integral over each coil by a seven-point Gaussian integration formula (ABRAMOwITZ and STEGUN, 1972).

(c) To investigate the influence of nonsystematic experimental errors, the $R D M$ value is determined where $F$ is obtained by the addition to $F_{r}$ of Gaussian noise having a zero mean value and a standard deviation (SD) which is proportional to the extremes of the field $F_{r}$. This proportional constant is called $G$.

(d) The influence on the field distribution of the models used for the volume conductor is estimated by means of simulation studies in which $F$ is based on either a model consisting of four realistically shaped compartments or four eccentric spheres. The shapes of the compartments are all taken from MR images of the same head (MEIJs et al., 1985). The radii and centres of
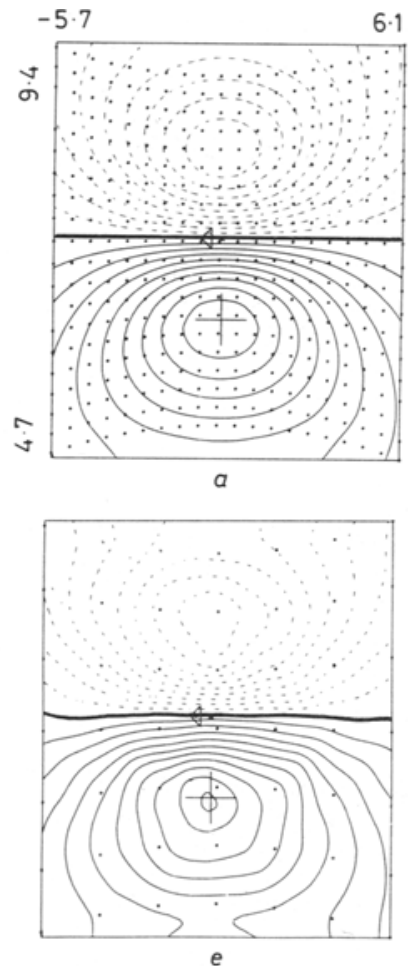

$\cdot 1$
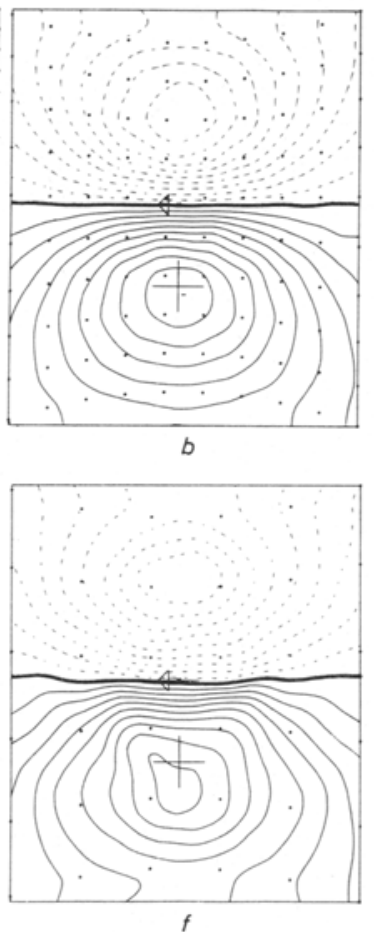
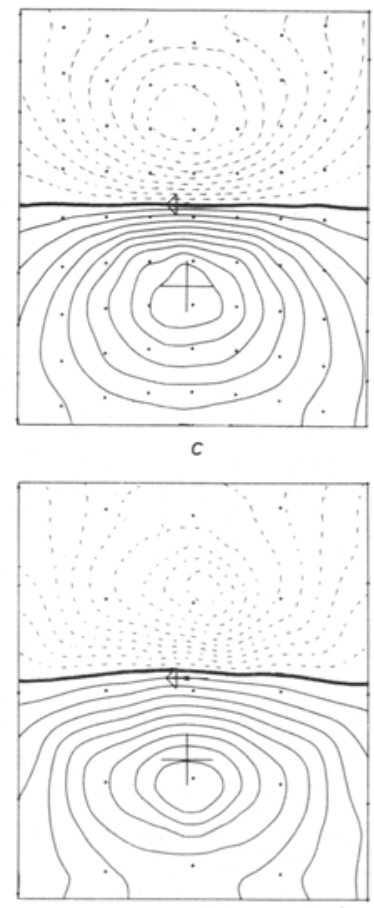

$g$
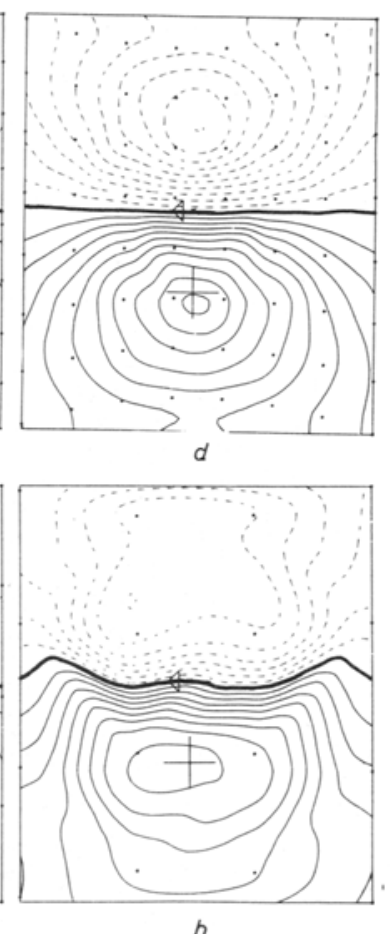

Fig. 1 Field patterns calculated from $N$ discrete grid points, represented by the small dots in the plots. The cross represents the projected location of the inion and the arrow represents the projection of the single current dipole on the recording surface. The dimensions of the maps are indicated on the first map of the series (in $\mathrm{cm}$ ). The first map (a) represents the reference field $F_{r}$, the number of grid points is $400(=20 \times 20)$. In the following maps of the series $(b-h)$ the number of grid points is reduced systematically from 100 $(=10 \times 10)$ in $(b)$ down to $16(=4 \times 4)$ in $(h)$ 
the eccentric spheres are deduced from the realistically shaped head model by optimally fitting a sphere to each compartment boundary in terms of a leastsquares fitting procedure (MeIJs and PETERS, 1987).

As an illustration, the compartment boundary representing the outside of the scalp in the realistically shaped model and the sphere fitting the scalp best in the visual cortex area is given in Fig. 2. The computation of the fields for both types of model is carried out by means of the boundary-element method as

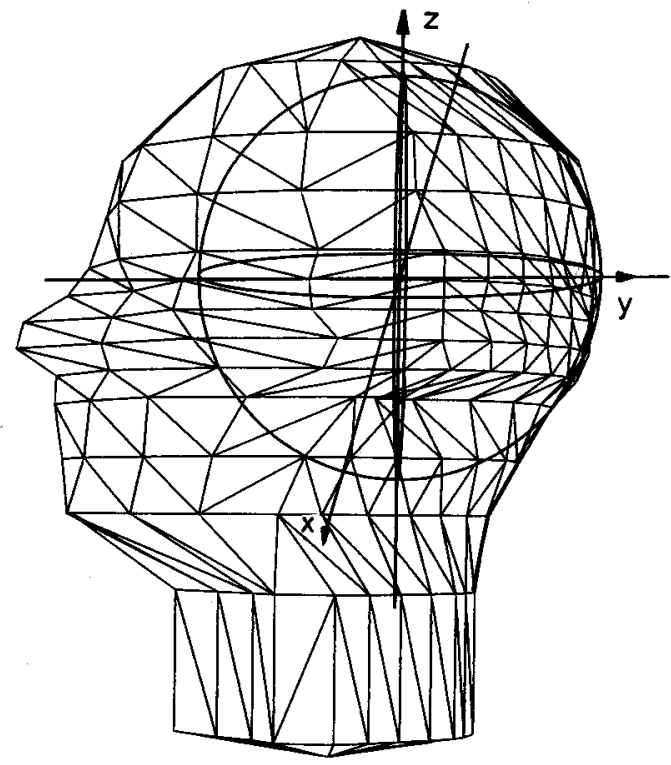

a described by LYNN and TIMLAKE (1968). The conductivities of the four compartments are restricted to low conductivity ratios in order to diminish numerical errors within the boundary-element method (MEIJs et al., 1987b). The values of scalp, skull, cerebrospinal fluid and brain tissue are taken to be, respectively, 0.33 , $0 \cdot 1,1 \cdot 0$ and $0.33 \mathrm{~S} \mathrm{~m}^{-1}$. A single current dipole, representing the model of the source, is oriented tangentially with respect to the concentric spheres model at about 50 distinct source locations throughout the

Fig. 2 Representation of the realistically shaped model of the head as seen from the left side and the back. Vertex points in the area of the visual cortex are used to compute the best fitting spheres. The sphere fitting the scalp is indicated in this figure as well. The cross in (b) represents the location of the inion

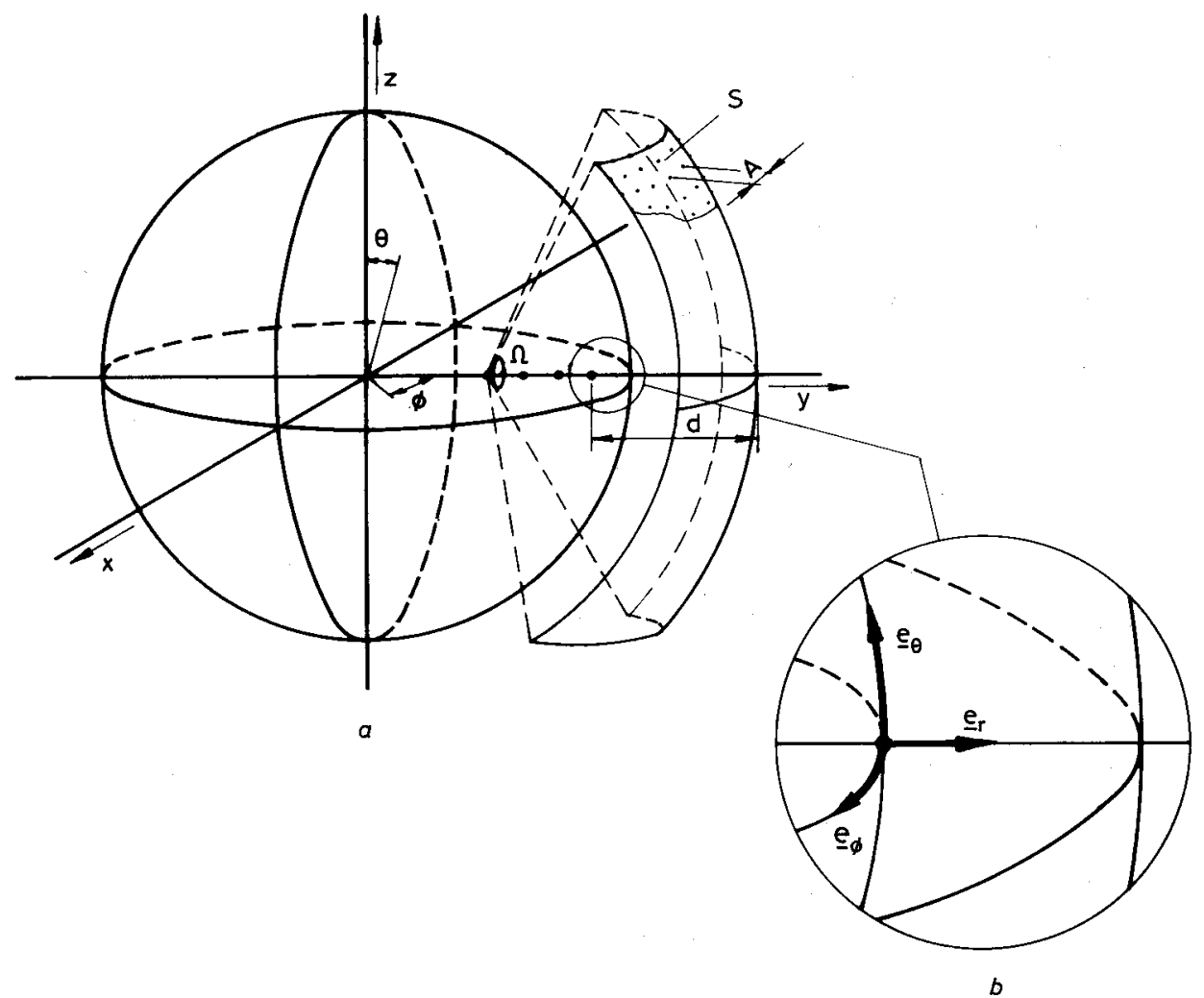

Fig. 3 (a) The orthonormal co-ordinate system $(x, y, z)$ is presented with respect to the outer sphere of the spherical models. This sphere coincides for both sphere models. The four dots on the positive $y$-axis indicate the source locations used. The distance between the single current dipolar source $p$ and the recording surface $S$ is defined as the source depth $d$. The sector of the recording sphere is bounded by the planes $x$ is constant and $z$ is constant. The distance between two neighbouring gridpoints is indicated by $A$. The solid angle $\Omega$ subtended by the sector $S$ of the recording sphere is a constant for each source. (b) The spherical co-ordinate system $\left(\boldsymbol{e}_{r}, \boldsymbol{e}_{\theta}, \boldsymbol{e}_{\phi}\right)$ is presented in the enlarged fragment 
occipital section of all three head models.

In all cases the reference field in the denominator of eqn. 1 is based on the concentric spheres model. The reference field in the numerator of eqn. 1 is based on either the concentric or on the realistically formed model of the head. This choice is made to be able to compare the fields based on the realistically shaped head and the eccentric spheres model by means of the RDM.

The models of the source considered are one single current dipole and a set of two current dipoles. The magnetic field distribution due to a single current dipole pointing in the asimuthal $e_{\phi}$ direction having a source strength $p$ which is located at $r$ is taken as the reference field. The influence of a displacement of the current dipole is studied by comparing the reference field with the field distribution generated by the same dipole at $r+l$. The displacements of $l$ studied are in the $e_{r}, e_{\theta}$ and $e_{\phi}$ directions (Fig. $3 b$ ). To estimate the influence of using two current dipoles instead of one, the reference field is compared with the field resulting from two current dipoles, each one pointing in the $e_{\phi}$ direction and having a dipole strength $p / 2$, which are located at $r+l / 2$ and $r-l / 2$. The directions of $l$ are chosen to be in the $e_{r}, e_{\theta}$ and $e_{\phi}$ directions.

\section{Results}

An impression of the pattern deformations due to the use of a limited number of grid points, using the bivariate interpolation technique (AкIMA, 1978), is given in Fig. 1. The distance between two neighbouring grid points is called $A$. For each source depth $d$, the same number of $N$ grid points is chosen on $S$; hence the quotient $A / d$ will be the same for all source depths. Values of the $R D M$ as a function of $N$ are given in Table 1 for a source depth of $32.8 \mathrm{~mm}$. For other source depths investigated (i.e. $42 \cdot 8$, 52.8 and $62.8 \mathrm{~mm}$ ), the $R D M$ values are comparable with those listed in Table 1. The fourth column of this table shows that in first-order approximation relation 2 holds:

$$
R D M=a \times\left(\frac{A}{d}\right)^{2}
$$

where $a$ is a constant which in first-order approximation is equal to 0.10 for all source depths except for the deepest source (its mean value is $0 \cdot 13$ ) and where only 16 grid points are taken.

The patterns given in Fig. 1 are practically the same if the effective magnetic field $B_{e f f}$ is taken instead of the normal component of the magnetic field. However, both $F$ and $F_{r}$ are decreased by an approximately constant factor. The value of this factor depends on the radii of the coils, the baseline of the gradiometer and the depth of the dipole. It is $\mathbf{0 . 7}$ for the gradiometer mentioned and a dipole with a source depth of $32.8 \mathrm{~mm}$. Consequently the values of the $R D M$ as listed in Table 2 show a sequence comparable to the one given in Table 1. Again the same relationship denoted by reln. 2 is found. However, the constant factor $a$ is slightly increased to a mean value of $0 \cdot 11$.

Evidently, the surface integral of $\left|F_{r}\right|$ over $S$ is linearly proportional to the value of the extremes of $F_{r}$. This ratio is found to be approximately 0.5 for the particular choice of $S$ and for the source depths mentioned. Therefore, if the Gaussian noise, added to $F_{r}$ to obtain $F$, has a zero mean and a standard deviation of $G$ times the extremes of $F_{r}$, the mean value of the $R D M$ will be approximately equal to $2 G$. The pattern of the map will be deformed at random.

Results of the $R D M$ values and the fields, using the eccentric spheres model and the realistically formed model, are given in Table 3 and Fig. 4.
Table 1 Values of both RDM and $R D M \times(d / A)^{2}$ due to the numerical computation of the surface integrals which are based on $N$ discrete values of the normal component of the magnetic field $B_{n}$. $A$ is the distance between two neighbouring measuring points, $d$ is the depth of the source. The mean value and the standard deviation of $R D M \times(d / A)^{2}$ are computed without using the values of the last row

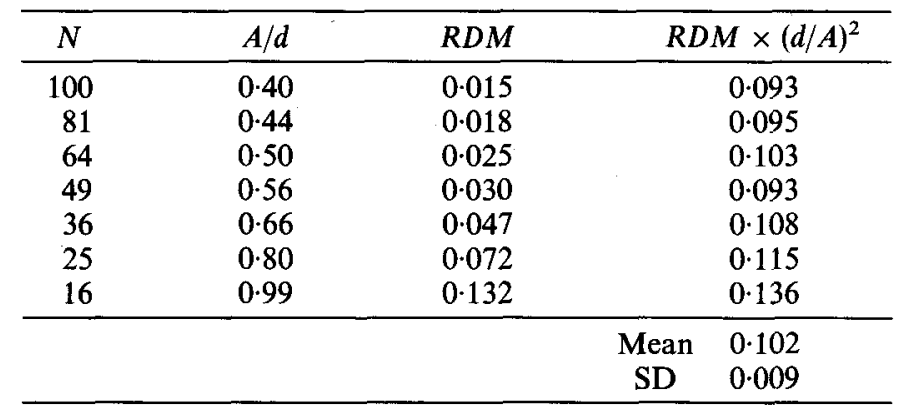

Table 2 The same as Table 1, now using the effective magnetic field $B_{\text {eff }}$ in the measurement points instead of the normal component of the field $B_{n}$

\begin{tabular}{|c|c|c|c|}
\hline$N$ & $A / d$ & $R D M$ & $R D M \times(d / A)^{2}$ \\
\hline 100 & 0.40 . & 0.016 & 0.099 \\
\hline 81 & 0.44 & 0.020 & $0 \cdot 104$ \\
\hline 64 & 0.50 & 0.027 & $0 \cdot 108$ \\
\hline 49 & 0.56 & 0.034 & $0 \cdot 108$ \\
\hline 36 & 0.66 & 0.053 & 0.122 \\
\hline 25 & 0.80 & 0.081 & 0.127 \\
\hline \multirow[t]{2}{*}{16} & 0.99 & $0 \cdot 139$ & $0 \cdot 142$ \\
\hline & & & $\begin{array}{c}\text { Mean } \\
\text { SD }\end{array}$ \\
\hline
\end{tabular}

Table 3 Values of the RDM quantifying the field differences using the concentric, the eccentric spheres model or the realistically formed head model for various source depths. $R D M(c, e)$ reflects the difference between the concentric and eccentric spheres model; $\operatorname{RDM}(c, r)$ reflects the difference between the concentric spheres model and the realistically shaped head model; and RDM(e,r) quantifies the differences between the eccentric spheres model and the realistic head model. For all three measures, the field distribution in the denominator is based on the concentric spheres model. The four sources are located on the line determined by the angular co-ordinates $(\theta, \phi)=(1.57,-0.40)$, (in rad, see Fig. 3)

\begin{tabular}{cccc}
\hline$d, \mathrm{~mm}$ & $R D M(c, e)$ & $R D M(c, r)$ & $R D M(e, r)$ \\
\hline 38 & 0.02 & 0.07 & 0.07 \\
43 & 0.03 & 0.09 & 0.08 \\
49 & 0.03 & 0.11 & 0.10 \\
56 & 0.04 & 0.14 & 0.13 \\
\hline
\end{tabular}

Table 4 Proportional constants $b$ between RDM and l listed as a function of the source depth $d$ and the direction in which the dipole is moved. The superscripts of the constants $b$ define the direction in which the dipole is moved. The proportional constants using an adapted source strength are denoted by $b^{r *}$. The standard deviation in each constant is less than 1 per cent of the mean value

\begin{tabular}{ccccc}
\hline$d$ & $b^{r}$ & $b^{r *}$ & $b^{\theta}$ & $b^{\phi}$ \\
\hline $\mathrm{mm}$ & $\mathrm{mm}^{-1}$ & $\mathrm{~mm}^{-1}$ & $\mathrm{~mm}^{-1}$ & $\mathrm{~mm}^{-1}$ \\
\hline 32.8 & 0.049 & 0.025 & 0.050 & 0.026 \\
42.8 & 0.044 & 0.018 & 0.043 & 0.021 \\
52.8 & 0.043 & 0.015 & 0.032 & 0.017 \\
62.8 & 0.049 & 0.018 & 0.034 & 0.015 \\
\hline
\end{tabular}

Analysis of the results due to a displacement $l$ of the current dipole showed that for the displacements of $l$ up to $6 \mathrm{~mm}$, the computed values of the $R D M$ can be approximated using the first term of the Taylor series expansion:

$$
R D M=b \times l
$$

where $b$ is a constant which depends on both the source location and the direction of the displacement. Values of $b$ are listed in Table 4. The strength of the dipole is kept 

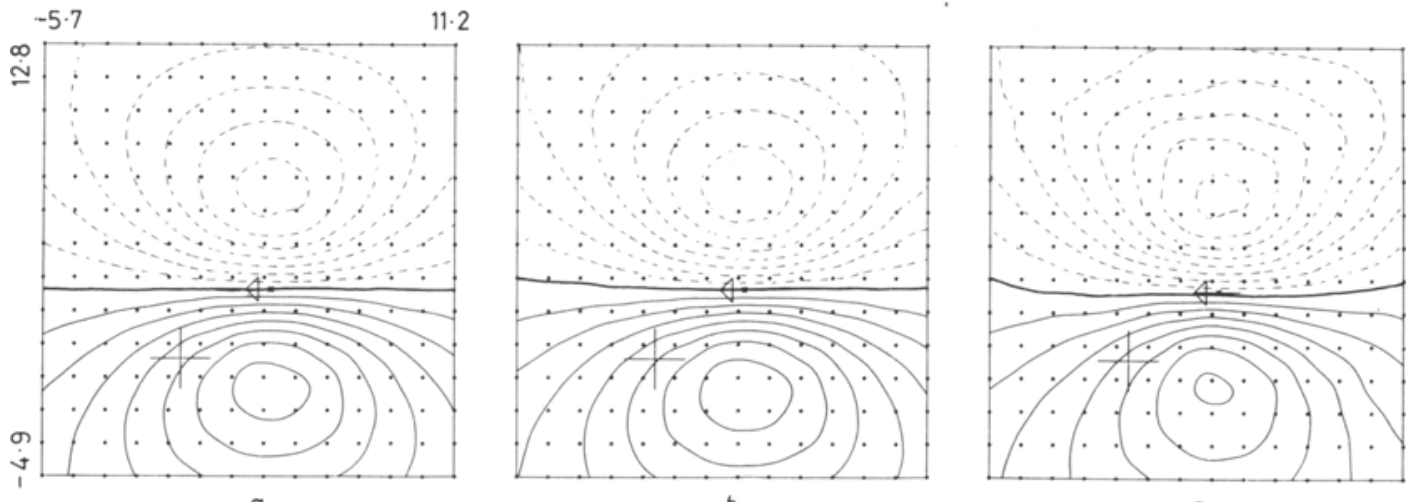

Fig. 4 Isomagnetic field maps based on three different volume conductor models. The symbols used in the plots are the same as in Fig. 1. In the first plot of the series, $(a)$, the reference field is presented. For (b) the eccentric spheres model of the head is used as the model of the volume conductor and for $(c)$ the realistically formed head model is used in the forward computation of the field pattern. This figure corresponds to the example given in the second row of Table 3

constant. However, when the dipole is displaced in the $e_{r}$ direction, the source strength can be so adapted that the extremes in the maps are kept constant. Using an adapted source strength gives values of the constant $b$ which are lower than those for which the strength is not adjusted. This is reflected in the second row of Table 4.

Resulting values of the $R D M$ due to splitting one single current dipole into two parallel dipoles separated at a distance $l$ showed that up to a distance $l$ of $30 \mathrm{~mm}$ the values of the $R D M$ can be described by the second-order term of the Taylor series expansion:

$$
R D M=c \times l^{2}
$$
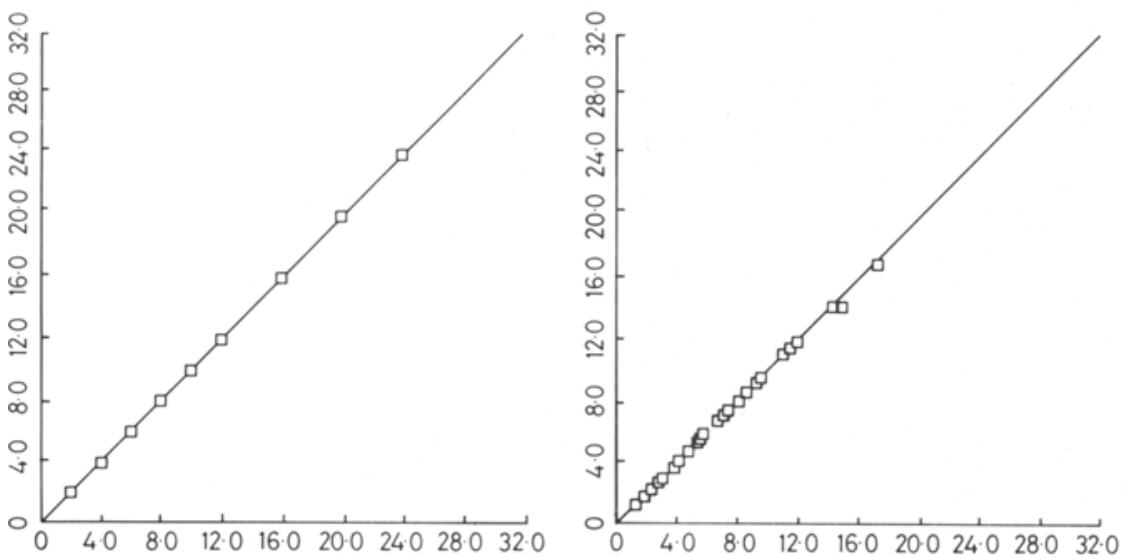

where $c$ is a constant which depends on both the source location and the direction of $l$. Fig. 5 shows that reln. 4 is appropriate for values of $l$ up to at least $30 \mathrm{~mm}$. Values of $c$ are listed in Table 5.

In Fig. $6 a$ the reference field generated by the single current dipole is given. In Fig. $6 b$ the source model used is a set of two dipoles pointing in the same direction as in Fig. $6 a$ but separated in the $e_{\theta}$ direction and in Fig. $6 c$ the two dipoles are separated in the $e_{\phi}$ direction.

\section{Discussion}

In Table 1 the values of the $R D M$ due to the use of a a

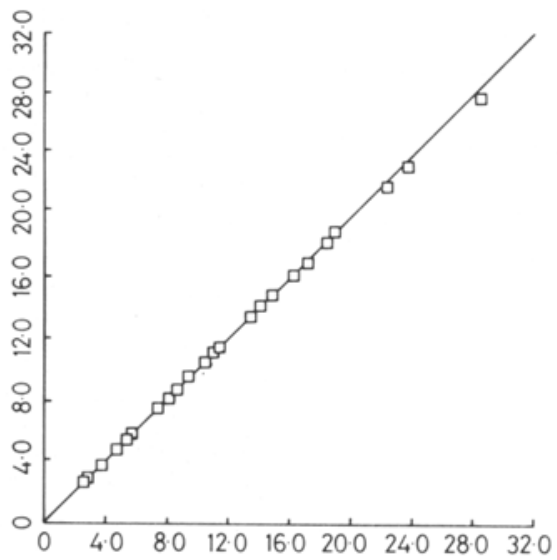

Fig. 5 Values of $\sqrt{ }(R D M / c)$ are given along the abscissa. Along the ordinate the distance 1 between the two sources is given. To compute the mean values of $c$, as listed in Table 5, six different values of $l$ are used at each source depth and for each direction of $l$. In (a)-(c) the direction of $l$ is, respectively, in the $e_{r}, e_{\theta}$ and $e_{\phi}$ direction. The lines $x=y$ drawn in each figure show that the second term of the Taylor series expansion (eqn. 4) gives an appropriate description of the RDM values

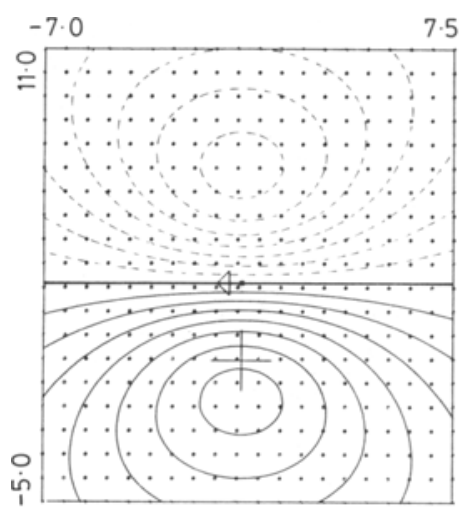

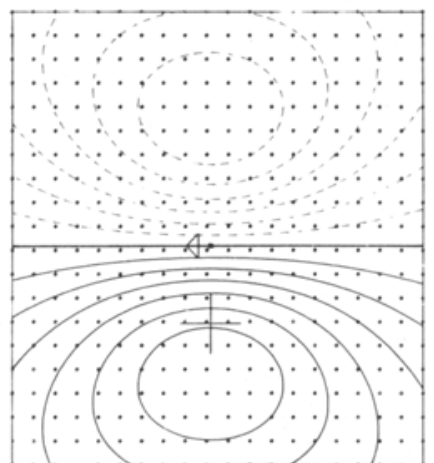

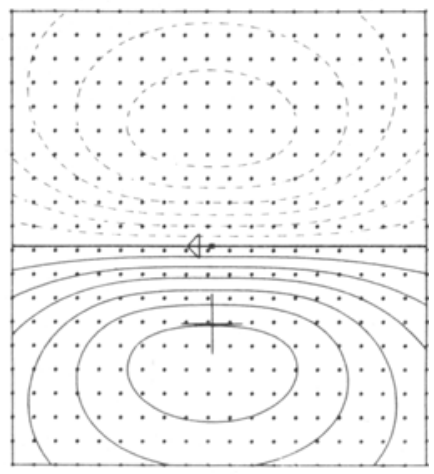

Fig. 6 The field distributions presented in these figures are due to (a) a single current dipole with a source depth of $42 \cdot 8 \mathrm{~mm},(b)$ two dipoles with the same source direction and the same source depth but separated in the $e_{\theta}$ direction with a distance of $14.2 \mathrm{~mm}$ and (c) two dipoles separated in the $e_{\phi}$ direction with a distance of $28.4 \mathrm{~mm}$. The values of the RDM are, respectively, 0.06 and 0.07 . The symbols used in the plots are the same as in Fig. 1 
Table 5 Proportional constants $c$ between the RDM and $l^{2}$ listed as a function of both the source depth and the direction in which the dipoles are separated. The superscripts of the constants reflect the direction in which the dipoles are separated. The standard deviation in each constant is less than 2 per cent of the mean values listed

\begin{tabular}{cccc}
\hline$d$ & $c^{r}$ & $c^{\theta}$ & $c^{\phi}$ \\
\hline $\mathrm{mm}$ & $\mathrm{mm}^{-2}$ & $\mathrm{~mm}^{-2}$ & $\mathrm{~mm}^{-2}$ \\
\hline 32.8 & $2.9 \times 10^{-4}$ & $3.8 \times 10^{-4}$ & $1.5 \times 10^{-4}$ \\
42.8 & $2.0 \times 10^{-4}$ & $2.9 \times 10^{-4}$ & $0.9 \times 10^{-4}$ \\
52.8 & $1.6 \times 10^{-4}$ & $2.7 \times 10^{-4}$ & $0.7 \times 10^{-4}$ \\
62.8 & $1.5 \times 10^{-4}$ & $3.3 \times 10^{-4}$ & $0.5 \times 10^{-4}$ \\
\hline
\end{tabular}

limited number of grid points are given for one source depth only. We found that the $R D M$ values for other source depths are the same for the same values of $N$. This can be understood by the following arguments: the angle between the two extremes of a dipolar pattern $\left(=2 \times \theta_{m}\right)$ depends on the source depth $d$ and the radius of the recording sphere $R$. This relationship can be derived and is given in eqn. 5 :

$$
\frac{3}{2 \cos \theta_{m}}-\frac{\cos \theta_{m}}{2}-1=\frac{d^{2}}{2(R-d) R}
$$

The depths $d$ of the sources taken in this study are confined to values between $33 \mathrm{~mm}$ and $63 \mathrm{~mm}$ under the recording sphere $S$ having a radius $R$ of $90 \mathrm{~mm}$. For this interval of the quotient $d / R$, the relationship between $d$ and $\theta_{m}$ can be approximated by a straight line (WILliamson and KaUfManN, 1980). The sector of the recording sphere is chosen such that it subtends the same solid angle with respect to the source for all sources. Since $\Omega$ is chosen constant for all source depths, it follows from $\Omega=S / d^{2}$ that the sides of the recording surface $S$ are linearly proportional to the source depth as well. This implies that for a certain value of $N$ the relative position of the grid points with respect to the dipole pattern will be comparable for all source depths in the range mentioned. For a homogeneous distribution of $N(=n \times n)$ grid points, the distance $A$ between two points is equal to $A=\sqrt{(S)} /$ ( $n-1)$, yielding $A / d=\sqrt{(\Omega)} /(n-1)$, which means that $A / d$ is a function of $N$ only. Combining this fact with eqn. 2 shows that the $R D M$ is a function of $N$ only.

There is no need to increase the number of grid points to reduce the $R D M$ value whenever the $R D M$ value due to other causes is much higher. Comparison of the orders of magnitudes of the $R D M$ s as given in this paper shows that an $R D M$ of 0.05 is relatively low. The minimum depth of a source under the recording sphere is about $30 \mathrm{~mm}$. The assumption that an $R D M$ value of 0.05 is low enough implies that the minimum distance between two neigh-

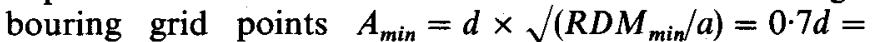
$20 \mathrm{~mm}$. This is in agreement with the findings of RoMANI and LEONI (1984), who considered the deformation of the continuous function due to the discrete sampling and concluded from this that no information in the measured signal was lost if the minimum distance between two neighbouring grid points met the same relationship, i.e. $A=0.7 d$.

If the effective magnetic field is used in inverse computations instead of the normal component of the magnetic field, the dipole strength will be underestimated although the location and the direction of the equivalent dipole will be estimated properly. This may explain the observed underestimation described by WEINBERG et al. (1985) of the source strength of the equivalent dipole. As both $F$ as well as $F_{r}$ are decreased by the same constant factor, the decrease of the fields due to the use of a gradiometer is not reflected in the value of the $R D M$.
It is clear that the influence of the eccentric spheres model on the magnetic field is smaller than that of the realistically shaped head model if the reference model is the concentric spheres model (columns 1 and 2 of Table 3). However, if the realistically shaped head is the reference field used in the numerator (columns 2 and 3), the values of the $R D M$ are comparable. These values are larger if the dipole lies deeper within the head.

If the Gaussian noise in the measurements is in the order of 25 per cent of the extremes of the field, which is an experimentally realistic figure, the $R D M$ due to this noise is approximately 0.5 for the dipolar patterns studied. Table 3 shows that the $R D M$ value is in the order of 0.1 if the compartment model of the head has realistically shaped boundaries instead of concentric spheres. Under these circumstances, the contribution of the volume currents cannot be distinguished. Similar interferences can be stated with respect to the resolution between two differing source locations by means of the MEG. If the difference between two locations of a dipole is $2 \mathrm{~mm}$, it follows from the combination of eqn. 3 and Table 4 that the $R D M$ value will be in the range $0 \cdot 03-0 \cdot 10$. This means that it will be difficult to decide which location is the best if the $R D M$ value due to noise is 0.5 .

It follows from Table 4 that if the dipoles are oriented in the $e_{\phi}$ direction, it is easier to resolve them if they are parallel than if they are in line with each other. This is in agreement with the concept of the so-called 'preferred direction' introduced by COHEN and CUFFIN (1983).

When two dipoles are separated by several $\mathrm{mm}$ (for example $7 \mathrm{~mm}$ ), most of the field (in this example 98 per cent) can be explained by taking one single current dipole in the middle as a substitute for the two. The value of the $R D M$ will then be smaller than 0.02. According to reln. 4 the value of the $R D M$ is proportional to the squared distance between the two dipoles. Therefore, if the distance between the two dipoles is $30 \mathrm{~mm}$, according to Table 5 , the $R D M$ will have a value which will be in the range $0.05-0.35$

The influence on the field of several other source models such as the 'in-line line source', the 'circular disk source' and the 'two opposed dipoles source' has been studied by Cuffin (1985). The residual error $F$ as used in Cuffin's study reflects the influence of the source models on the field. This residual error is defined as the normalised sum of the squared differences of two fields in corresponding grid points, and consequently it differs only slightly from the $R D M$. Since Cuffin found that the $F$-values for other source models are comparable with the values found for the 'two dipoles' source model used in this study, there is no need to repeat the investigation of the other source models.

The comparison of the $R D M$ s given above is based on a subjective way of weighting. However, the $R D M$ gives quantitative information on the field pattern deformations in the case where dependencies on one single parameter are studied.

\section{Conclusions}

The relative difference measure $R D M$ can be used to quantify differences observed between a map showing an ideal dipole pattern and a simulated one for which model or measurement parameters are changed one at a time. We do not claim that the $R D M$ is superior in some specific sense, but it is a very useful tool for obtaining a quantitative comparison of field distributions. However, as well as the calculation of the $R D M$ a visual inspection of the field pattern is important. The dipolar pattern of an isofield 
map which is influenced by noise shows deformations which are clearly detectable by visual inspection. On the other hand, comparison of Figs. $5 b$ and $c$ gives an example in which the dipole pattern is conserved in such a way that visual quantification is difficult although the $R D M$ value changes substantially.

It is clearly impossible to distinguish between two different values of a parameter or between two different models if this difference gives rise to an $R D M$ value of about 0.05 , because the $R D M$ originating from arbitrary errors in the measurements has a value of about 0.5 . Therefore the values of the $R D M$ are very useful for determining which change in a parameter or model can still be detected by means of experimentally obtained magnetoencephalograms.

An analogous error measurement is also applicable in the analysis of EEGs.

Acknowledgment-We would like to thank Professor Dr A. van Oosterom (University of Nijmegen) for his valuable remarks and stimulating discussions on this work.

\section{References}

ABramowitz, M. and Stegun, I. A. (1972) Handbook of mathematical functions. Dover Publ. Inc.

AKIMA, H. (1978) A method of bivariate interpolation and smooth surface fitting for irregular distributed data points. ACM Trans. on Math. Software, 4, 148-159.

COHEN, D. and CuFfin, B. N. (1983) Demonstration of useful differences between magnetoencephalogram and electroencephalogram. Electroenceph. Clin. Neurophysiol., 56, 38-51.

CuFfin, B. N. (1985) A comparison of moving dipole inverse solutions using EEG's and MEG's. IEEE Trans., BME-32, 905-910.

GrynszPan, F. and Geselowitz, D. B. (1973) Model studies of the magnetocardiogram. Biophys. J., 13, 911-925.

LeHMANN, H. P. (1983) Signal processing. In Biomagnetism: an interdisciplinary approach. WILliamson, S. J., RomaNI, G. L., Kaufman, L. and Modena, I. (Eds.), Plenum Press, New York, London, 591-624.

LynN, M. S. and Timlake, W. P. (1968) The use of multiple deflations in the numerical solution of singular systems of equations with applications to potential theory. Siam. $J$. Numer. Anal., 5, 303-322.

MacAulay, C. E., Stroink, G. and Horacek, B. M. (1985) Analyses of MCG spatial maps during the PR-interval. Med. \& Biol. Eng. \& Comput., 23, Suppl., Part 2, 1479-1480.

Meiss, J. W. H., Peters, M. J. and van Oosterom, A. (1985) Computation of MEGs and EEGs using a realistically shaped multi-compartment model of the head. Ibid., 23, Suppl., Part 1, 36-37.

MeiJs, J. W. H., Bosch, F. G. C., Peters, M. J. and Lopes DA Silva, F. H. (1987a) On the magnetic field distribution generated by a dipolar current source situated in a realistically shaped compartment model of the head. Electroenceph. Clin. Neurophysiol., 66, 286-298.

Meiss, J. W. H., Boom, H. B. K., Peters, M. J. and van OosTEROM, A. (1987b) Application of the Richardson extrapolation in simulation studies of EEGs. Med. \& Biol. Eng. \& Comput., 25, 222-226.

Meiss, J. W. H. and Peters, M. J. (1987) The EEG and MEG, using a model of eccentric spheres to describe the head. IEEE Trans., BME-34, (12).
Romani, G. L. and LeONI, R. (1984) Localization of cerebral sources by neuromagnetic measurements. In Biomagnetism: applications and theory. WeinberG, H., Stroink, G. and Katila, T. (Eds.), Pergamon Press, Vancouver, 205-220.

SToK, C. J. (1987) EEG/MEG single dipole source estimation. IEEE Trans., BME-34, 289-296.

Weinberg, H., Brickett, P., Coolsma, F. and BafF, M. (1985) Magnetic localisation of intracranial dipoles: simulations with a physical model. Electroenceph. Clin. Neurophysiol., 64, 159170

Williamson, S. J. and Kaufman, L. (1980) Magnetic fields of the cerebral cortex. In Biomagnetism. ERNE, S. N., HaHLBOHM, H. D. and LueBBIG, H. (Eds.), De Gruyter, Berlin, 353-402.

\section{Authors' biographies}

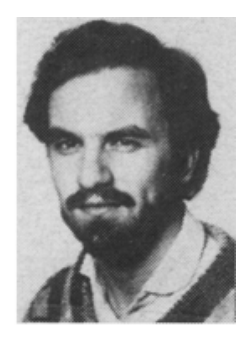

Hans Meijs was born in Heerlen, the Netherlands in 1959. He received the M.Sc. degree in Physics from the University of Eindhoven in 1984. His M.Sc. thesis was on the composition of a realistically shaped multicompartment model of the head. Since 1984 he has been with the Biomedical Engineering Division of Twente University preparing a Ph.D. thesis on MEG and EEG simulation and measurements.

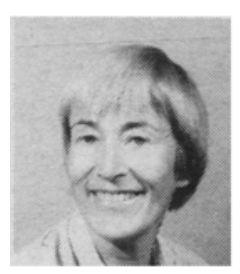

Maria Peters graduated in Physics at the University of Leiden. She received her Ph.D. at Twente University. Her thesis was on magnetocardiography. Her current research interests include the experimental and theoretical development of biomagnetism.

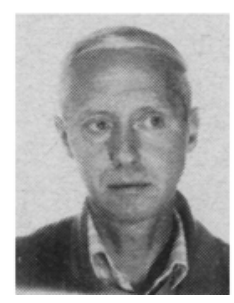

Herman Boom was trained as a medical physicist at the University of Utrecht, where he received his $\mathrm{Ph} . \mathrm{D}$. in 1971. He joined the departments of Medical Physics and Medical Physiology, where he was engaged in research in the field of cardiac mechanics and taught Physiology and Biophysics. Since 1976 he has occupied the chair of Medical Electronics in the Electrical Engineering department of the University of Twente. His research interests are cardiovascular system dynamics, bioelectricity and rehabilitation technology.

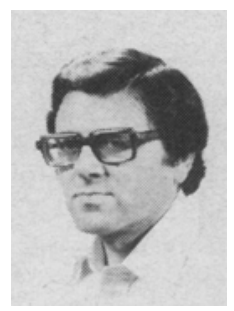

Fernando Lopes da Silva was born in 1935 and received his medical degree from the University of Lisbon in 1959, studied postgraduate Engineering and Physics for Physiologists at Imperial College, London, and received his Ph.D. from the University of Utrecht in 1970. $\mathrm{He}$ has held academic posts and professorships in the UK, The Netherlands and Portugal. He is currently Professor in General Animal Physiology at the University of Amsterdam. His main research interests are in the field of synaptic plasticity, epileptogenesis and biophysical aspects of electric and magnetic signals in the central nervous system. 